\title{
Synthesis of control laws of electromechanical systems under polynomial approximation of characteristics of nonlinear elements
}

\author{
Vladislav Shishlakov*, Elizaveta Vataeva, Natalia Reshetnikova and Dmitriy Shishlakov \\ SUAI, Department of Electromechanics and Robotics, 190000, St. Petersburg, Bolshaya Morskaya Str., 67, Russia
}

\begin{abstract}
The paper presents the solution of the problem of synthesis of the laws of control of electromechanical automatic control system under polynomial approximation. As a mathematical apparatus of the decision the generalized Galerkin's method is applied. This method is extended to a new class of objects with nonlinear characteristics approximated polynomially.
\end{abstract}

\section{Introduction}

Providing the set indicators of quality of work of the nonlinear systems of automatic control (ACS) in the transitional modes represents very difficult task which correct solution is in many respects connected with creation of adequate mathematical model.

As the elements and devices having nonlinear characteristics enter into structure of electromechanical systems and complexes, when developing mathematical models, degree of their adequacy is directly connected with the accepted way of approximation of not linearities [1-3].

The most widespread method of approximation of nonlinear characteristics is piecewise and linear approximation. However, the models received thus not always can with sufficient degree of accuracy reproduce nonlinear characteristics, especially, if it is about extreme characteristics such as mechanical characteristic of the asynchronous engine or dependence of coefficient of coupling on the size of relative slipping. Similar dependence by means of piecewise and linear approximation can be reproduced rather precisely at very large number of piecewise line sections. Reduction of number of piecewise line sections for approximation allows to receive quickly enough result, however the error of calculation can be unacceptably high. At the same time complication of the approximating expressions for nonlinear characteristics can create considerable difficulties at a research as dynamic properties of nonlinear control systems in itself are rather difficult.

Therefore it is advisable to use polynomial (algebraic) approximation in such cases which unlike the theory of automatic control is very widely used and applied in electrical engineering. In this case, the approximating polynomial is written in the form

$$
y(x)=y\left(x_{0}\right)+a_{1}\left(x-x_{0}\right)+a_{2}\left(x-x_{0}\right)^{2}+K+a_{n}\left(x-x_{0}\right)^{n}(1)
$$

where coefficients of $a_{1}, a_{2} \ldots, a_{n}$ are defined by expressions

$$
\begin{aligned}
& a_{1}=\left(\frac{d v}{d x}\right)_{x=x_{0}} \\
& a_{2}=\frac{1}{2 !}\left(\frac{d^{2} v}{d x^{2}}\right)_{x=x_{0}} \\
& a_{n}=\frac{1}{n !}\left(\frac{d^{n} v}{d x^{n}}\right)_{x=x_{0}} ; K
\end{aligned}
$$

Approximation by a power polynomial consists in finding the coefficients of the series $a_{1}, a_{2} \ldots, a_{n}$. At the set characteristic form these coefficients significantly depend on the choice of a working point and also on width of the used site of the characteristic.

An effective method for solving the problem of synthesizing the parameters of the control law for electromechanical and robotic systems and complexes is the generalized Galerkin's method allowing to solve from uniform mathematical, methodological and algorithmic positions similar problems for ACS of a wide class which dynamic properties are described by the nonlinear differential equations randomly of a high order.

\section{Mathematical description of the synthesis problem}

From a mathematical point of view, this approach makes it possible to approximately solve a nonlinear differential equation of arbitrary form of any order, describing the dynamics of the automatic control system $[4,5]$.

$$
\begin{aligned}
& Q\left(c_{k}, p\right) x(t)+R\left(c_{k}, p\right) y(t)=S\left(c_{k}, p\right) f(t) ; \\
& y(t)=F[x(t), \dot{x}(t)],
\end{aligned}
$$

\footnotetext{
* Corresponding author: kaf31guap@gmail.com
} 
where $x(t)$ - coordinate at the input of the non-linear link, relative to which the equation of motion of the synthesized ACS is written; $f(t)$ - the external input; $y(t)=F[x(t), \dot{x}(t)]-$ a nonlinear function representing a relationship approximating the static or dynamic nonlinear characteristic of an ACS element or device.

Polynomials of the differentiation operator $p$ with real constant coefficients of powers of $n, u, v$ respectively, $c_{k}$ are the desired parameters of the control operator.

In the course of the solution, it is required to find the values of unknown parameters that will provide the system with specified quality indicators of its operation during the transition from one steady state to another.

As a rule, the problem is solved with constraints on the values of the coefficients of the differential equation (which are functions of the parameters of the control operator), while the solution may be close to the given, but not accurately reproduce it. This is due to the fact that in any ACS there is an unchangeable part (the executive motor, the transmission mechanism, the mismatch meters, etc.), the transmission coefficients and the time constants of the elements of which are given. In addition, the parameters of the management operator are constrained by the possibility of their technical implementation

$$
\begin{aligned}
& c_{k}^{-} \leq c_{k} \leq c_{k}^{+} \\
& k=1,2, K, m,
\end{aligned}
$$

where $c_{k}^{+}-$the maximum permissible value of the variable parameters; $c_{k}^{-}-$the minimum permissible value of the variable parameters.

And also by the restriction on the roughness of the solution found for given deviations of the parameters from the values found, which is related to the technological spread of the element base

$$
\Delta=\frac{\delta c_{k}}{c_{k}} \leq \Delta^{0}
$$

where $\Delta^{0}$ is a given value of system roughness; $\delta c_{k}-$ variations of parameters within which the system is stable.

When determining the parameters of the control operator of a nonlinear automatic control system in the first approximation, the desired software motion is given in the form of a solution of a linear differential equation.

Since the design of automatic control systems, regulated indicators, are usually indicators of the quality of the transient process, then. there must be a one-to-one correspondence between these indicators and the parameters of the desired program motion (the damping coefficients and the natural vibration frequencies of its components).

Such a correspondence is defined for linear differential equations of the first and second orders. So the attenuation coefficient $\alpha$ is related to the duration of the transient process $T_{n \cdot n}$ by the relation $[4,5]$

$$
\alpha=\frac{3 \div 4}{T_{\text {пा }}}
$$

The solution of a second-order linear differential equation has the form

$$
x^{0}(t)=\left[x_{y}+H^{*} \cos \left(\beta t-\varphi_{0}\right) e^{-\alpha t}\right] 1(t)
$$

where $x_{y}$ - the value of the desired process $x^{0}(t)$ at $t=\infty$; and $H^{*}$ and $\varphi_{0}$ are defined by relations of the form

$$
\begin{aligned}
& H^{*}=\sqrt{\left(x_{0}-x_{y}\right)^{2}+\left[\frac{\alpha\left(x_{0}-x_{y}\right)+\dot{x}_{0}}{\beta}\right]} \\
& \varphi_{0}=\operatorname{arctg}\left[\frac{\alpha\left(x_{0}-x_{y}\right)+\dot{x}_{0}}{\beta\left(x_{0}-x_{y}\right)}\right]
\end{aligned}
$$

here $x_{0}, \dot{x}_{0}-$ the initial values of the coordinate under consideration, with respect to which the equation of motion of the synthesized ACS is recorded and it's derivative, respectively, at time $t=+0$.

The oscillation of the process $\mu$ is related to overshoot $\sigma$ which in turn determines the natural oscillation frequency $\beta$. Thus, in the particular case (when $=0)$, we have

$$
\sigma_{\mathrm{m}}=\frac{\mu}{\sqrt{\mu^{2}+1}} e^{-\frac{\pi}{\mu}}
$$

Thus, proceeding from the required values of the performance indicators of the ACS in the transient mode (time of the transient and overshoot), it is possible to determine the values of the parameters of the program motion of the form (7) that provide them.

For more complex software movements (for example, the third fourth order), some of the process parameters must be assigned, and the rest must be determined, as shown in [5].

After the desired program motion (the solution of the nonlinear differential equation) is specified and its parameters are determined, a discrepancy is formed

$$
\begin{aligned}
& \Psi\left(c_{k}, t\right)=Q\left(c_{k}, D\right) x^{\circ}(t)+ \\
& +R\left(c_{k}, D\right) F\left[x^{\circ}(t), D\left\{x^{\circ}(t)\right\}\right]- \\
& -S\left(c_{k}, D\right) f(t),
\end{aligned}
$$

where $x^{0}(t)$ - the desired software motion (the solution imposed on the nonlinear differential equation), $D-$ the operator of generalized differentiation.

The values of the unknown parameters $c_{k}$ are determined from the orthogonality condition of the discrepancy (10) to the coordinate functions

$$
\begin{aligned}
& \int_{0}^{\infty} \psi\left(c_{k}, t\right) \varphi_{q}(t) d t=0 \\
& k, q=1,2, K, m
\end{aligned}
$$


where $\varphi_{q}(t)$ - system of $m$ continuously differentiable linearly independent coordinate functions

$$
\varphi_{1}(t), \varphi_{2}(t), K, \varphi_{q}(t), K, \varphi_{m}(t)
$$

Coordinate functions should be a complete system of functions. In this method of solving a nonlinear differential equation of general form, real exponents are used (in contrast to the Laplace transform, in which complex exponentials are used)

$$
e^{-\rho_{1} t}, e^{-\rho_{2} t}, K, e^{-\rho_{q} t}, K, e^{-\rho_{m} t}, q=1,2, K, m
$$

The experience of solving practical problems shows [47] that in order to best approximate the desired program motion $x^{o}(t)$ to the actual process occurring in the system with synthesized parameters, the attenuation coefficient $\rho_{1}$ of the coordinate functions is expediently chosen in the form

$$
\rho_{1}=\alpha
$$

where $\alpha$ - the attenuation coefficient of the component of the desired program motion, which has the greatest influence on the duration of the transient process.

The remaining attenuation coefficients of the series $p_{m-1}$ should be chosen in the form of a geometric progression (with the denominator of the progression $r=2$ ), that is

$$
\rho_{q}=\rho_{1} r^{q-1}=\rho_{1} 2^{q-1}, q=1,2, K, m
$$

this provides a shorter fade time for each of the $m-1$ exponentials compared to the decay time of the first coordinate function.

The solution of equation (11) leads to the following system of algebraic equations

$$
\begin{gathered}
\sum_{i=0}^{n} a_{i}\left(c_{k}\right) A_{q i}+\sum_{i=0}^{u} b_{i}\left(c_{k}\right) B_{q i}-\sum_{i=0}^{v} e_{i}\left(c_{k}\right) C_{q i}=0, \\
q=1,2, \ldots, m,
\end{gathered}
$$

where

$$
\begin{aligned}
& A_{q i}=\int_{0}^{\infty} D^{i}\left\{x^{\circ}(t)\right\} e^{-\rho q^{t}} d t=A_{q} \rho^{i-1}, \\
& i=0,1, \ldots, n, \\
& B_{q i}=\int_{0}^{\infty} D^{i}\left\{F\left[x^{\circ}(t), \dot{x}^{\circ}(t)\right]\right\} e^{-\rho} q^{t} d t=B q \rho^{i-1}, \\
& i=0,1, \ldots, u, \\
& C_{q i}=\int_{0}^{\infty} D^{i}\{f(t)\} e^{-\rho_{q} t} d t=C_{q} \rho^{i-1}, \\
& i=0,1, \ldots, v .
\end{aligned}
$$

In [4-6] the method of calculating Galerkin integrals is considered in detail and recurrent analytical expressions $A_{q}, B_{q}, C_{q}$ for differential equations with various nonlinear characteristics are given for their piecewise linear and algebraic approximations for various types of program motions.

Solving the system of $\mathrm{m}$ algebraic equations (16), we determine the values of the variable parameters of the control operator, and, consequently, the coefficients of the nonlinear differential equation of motion of the ACS.

However, the problem is solved with limitations on the values of the required parameters imposed on the basis of the possibility of their technical implementation, limitations on the stability and roughness of the ACS with synthesized parameters, and also because, as a rule, there is a nonlinear relationship between the varied parameters, then the strict equality (16) will not be performed.

Therefore, the definition of the parameters of the control operator providing a given solution of a nonlinear differential equation in the computational plan is a non-linear programming problem with an objective function constructed on the basis of equations (16) and having the form

$$
J=\sum_{q=1}^{m}\left\{\int_{0}^{\infty} \psi\left(c_{k}, t\right) \varphi_{q}(t) d t\right\}^{2}, \min _{c_{k}} J \rightarrow 0,
$$

The optimum of which is determined under the constraints noted above using the known methods for finding the extremum of a functional.

To develop the generalized Galerkin method for a new class of approximating nonlinear characteristics of functions, it is required to determine the values of the integrals $B_{q i}$ on a family of elementary functions.

That is, for the solution of a problem of synthesis of ACS at polynomial approximation of characteristics it is required to calculate integral

$$
\begin{aligned}
& B_{q i}=\int_{0}^{\infty} D^{i}\left\{F\left[x^{0}(t)\right]\right\} e^{-\rho_{q} t} d t= \\
& =\int_{0}^{\infty} D^{i}\left\{\sum_{g=0}^{l} z_{g}\left(x^{0}(t)\right)^{g} 1(t)\right\} e^{-\rho_{q} t} d t
\end{aligned}
$$

where

$$
F\left[x^{0}(t)\right]=\sum_{g=1}^{l} F_{g}\left[x^{0}(t)\right]=\sum_{g=0}^{l} z_{g}\left(x^{0}(t)\right)^{g}, g=0,1, \ldots, l
$$

what represents distribution of the principle of equivalent transformations [5, 6] on nonlinear characteristics at their analytical approximation and significantly simplifies calculation of integrals.

The experience of solving practical problems in the synthesis of linear and nonlinear mathematical models of control systems by the generalized Galerkin method shows that it is sufficient to take the solution of a linear differential equation of no higher than second order $[5,7]$ which, as a rule, is less than the order of the differential equation describing the motion of the synthesized system. This provision seems to be completely justified, since the problem of synthesis, rather than identification, is being solved. Therefore, emphasizing the higher harmonic components in the program movement, whose influence they seek to reduce, has no practical meaning. 
In addition, in the design of automatic control systems, regulated indicators, usually indicators of the quality of the transient process. Therefore, there must be a one-to-one correspondence between these parameters and the parameters of the desired program motion (damping coefficients and natural frequencies of oscillations of its components), which is defined for linear differential equations of the first and second orders.

As a result of the calculations, we obtain

$$
B_{q i}=\int_{0}^{\infty} D^{i}\left\{F\left[x^{0}(t)\right]\right\} e^{-\rho_{q} t} d t=B_{q} \rho_{q}^{i-1}, i=0,1, \ldots, u,
$$

where the ratios defining $B_{q i}$ are calculated for process

$$
x^{0}(t)=\left[H^{*} e^{-\alpha t} \cos \left(\beta t-\varphi_{0}\right)\right] 1(t)
$$

corresponding to the recording of the equation of motion of the system relative to the input and process

$$
x^{0}(t)=\left(x_{y}-H^{*} e^{-\alpha t} \cos \left(\beta t-\varphi_{0}\right)\right) 1(t)
$$

corresponding to the recording of the equation of motion of the system relative to the error signal.

We get:

- for odd degree

$$
B_{q}=\sum_{g=0}^{l} a_{g} \sum_{k=0}^{g} H^{* k} C_{g}^{k}\left\{\begin{array}{c}
\frac{k}{2^{k-1}\left(k \alpha+\rho_{q}\right)}\left[1-\left(k-2 E\left(\frac{k}{2}\right)\right)\right]+ \\
\frac{\left(k \alpha+\rho_{q}\right) \cos \left(k \varphi_{0}\right)+k \beta \sin \left(k \varphi_{0}\right)}{2^{k-1}\left[\left(k \alpha+\rho_{q}\right)^{2}+(k \beta)^{2}\right]} \\
\frac{k\left[\begin{array}{l}
\left.\left(k \alpha+\rho_{q}\right) \cos \left((k-2) \varphi_{0}\right)+\right] \\
(k-2) \beta \sin \left((k-2) \varphi_{0}\right)
\end{array}\right]}{2^{k-1}\left[\left(k \alpha+\rho_{q}\right)^{2}+((k-2) \beta)^{2}\right]}
\end{array}\right\}
$$

- for an even degree

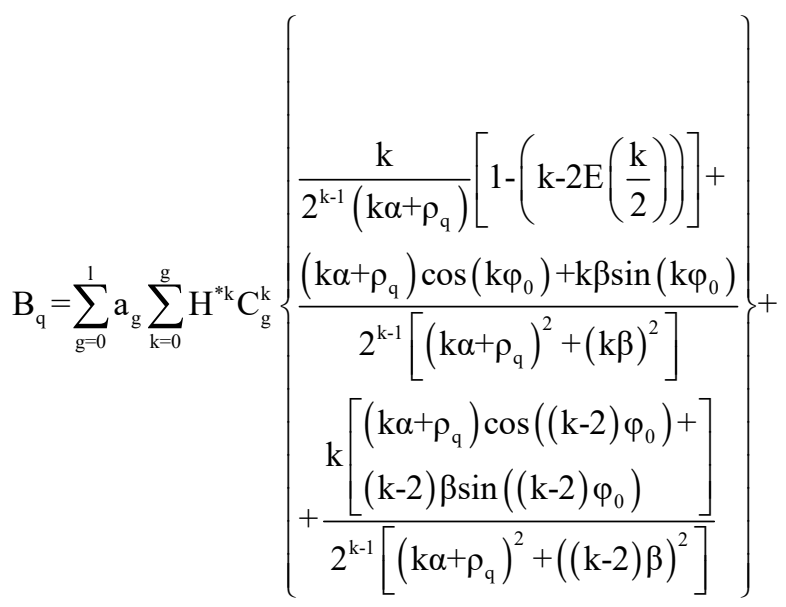

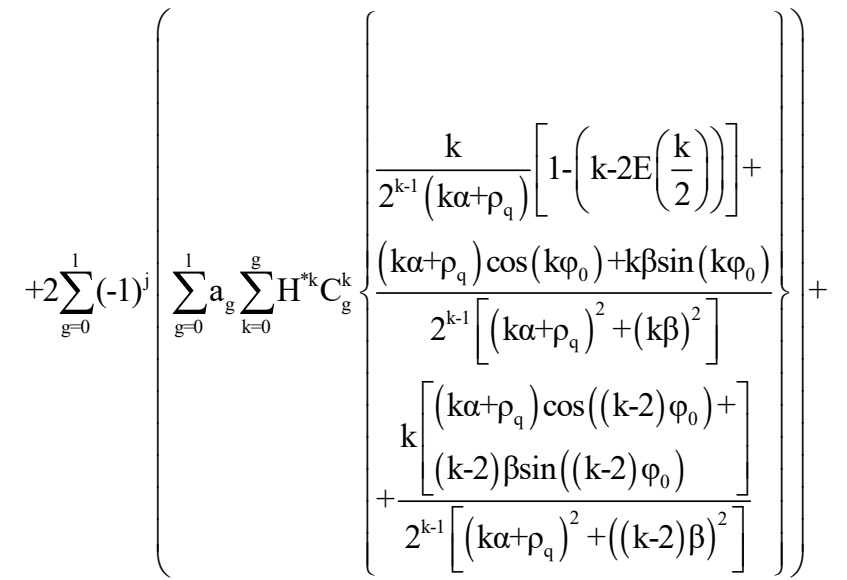

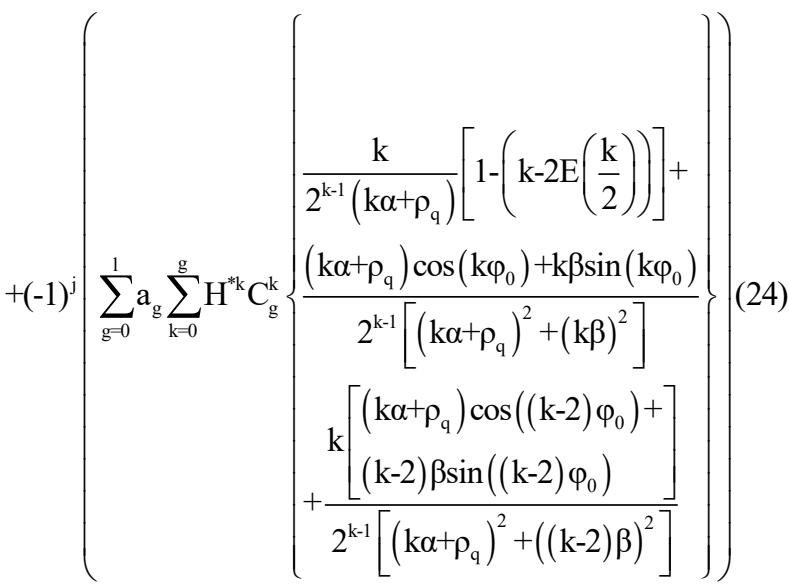

here $E$ is the integer part of a number.

Summarizing the expressions (23) and (24), we obtain

$$
B_{q}=\sum_{g=0}^{l} a_{g} \sum_{k=0}^{g} x_{y}^{g-k} H^{* k}(-1)^{k} C_{g}^{k}\left\{\begin{array}{c}
\frac{k}{2^{k-1}\left(k \alpha+\rho_{q}\right)}\left[1-\left(k-2 E\left(\frac{k}{2}\right)\right)\right]+ \\
\frac{\left(k \alpha+\rho_{q}\right) \cos \left(k \varphi_{0}\right)+k \beta \sin \left(k \varphi_{0}\right)}{2^{k-1}\left[\left(k \alpha+\rho_{q}\right)^{2}+(k \beta)^{2}\right]} \\
k\left[\begin{array}{l}
\left(k \alpha+\rho_{q}\right) \cos \left((k-2) \varphi_{0}\right)+ \\
(k-2) \beta \sin \left((k-2) \varphi_{0}\right)
\end{array}\right] \\
+\frac{2^{k-1}\left[\left(k \alpha+\rho_{q}\right)^{2}+((k-2) \beta)^{2}\right]}{}
\end{array}\right\}
$$

\section{Conclusion}

Thus, the recurrence relations defining the Galerkin integrals $B_{q i}$ for polynomial nonlinear characteristics make it possible to apply the method for solving the problem of synthesis of the control laws of electromechanical and robotic systems and complexes for a given approximation of the characteristics of nonlinear element. 


\section{References}

1. L. Bessonov, Theoretical fundamentals of electrical engineering: Electric circuits (Higer school, Moscow, 1978)

2. K. Polivanova, D. Zhukhovitsky, I. Negnevitsky Theoretical bases of electrical (Energy, Moscow, 1972)

3. G. Zeveke, P. Ionkin, A. Netushil, S. Strakhov, Fundamentals of the theory of chains (Energoatomizdat, Moscow, 1989)

4. V. Shishlakov, Synthesis of nonlinear automatic control system with various types of modulation: monograph (SPbSUAI, SPb, 1999)

5. A. Nikitin, V. Shishlakov, Parametric synthesis of nonlinear automatic control system: monograph (SPbSUAI, SPb, 2003)

6. V. Shishlakov, S. Tsvetkov, D. Shishlakov, Synthesis of the parameters of continuous and pulsed multiply connected automatic control system: monograph (SPbSUAI, SPb, 2009)

7. V. Shishlakov, A. Shishlakov, S. Timofeev, Synthesis of automatic control system for various types of approximation of nonlinear characteristics : theory and practise (SPbSUAI, SPb, 2017)

8. M.V. Burakov, V.F. Shyshlakov, SPIIRAS Proceedings, 51(2), 60-77 (2017). doi:10.15622/sp.51.3 